\title{
A manualised in-patient programme for young people with anorexia based on NICE guidance
}

\section{AIMS AND METHOD}

The Rainbow programme was introduced in January 2004 as a structured in-patient treatment for anorexia. There were two strands to the programme evaluation: a notes review of a cohort of in-patients and a series

\author{
of questionnaires to users, carers \\ and staff. \\ RESULTS \\ There was an improvement in \\ adherence to the National Institute \\ for Health and Clinical Excellence \\ guidelines and $94 \%$ of staff
}

\author{
responders found the Rainbow \\ programme framework useful. \\ CLINICAL IMPLICATIONS \\ The Rainbow programme improved \\ the level of care provided. There is \\ scope for improvement in the distri- \\ bution of the programme manual.
}

Anorexia nervosa remains an illness with significant mortality and morbidity, especially in adolescents (Gowers et al, 2000). Hospitalisation has been criticised (Gowers et al 2000), but is still needed, especially where critical concerns for physical well-being are present. The evidence base for effective interventions in children and adolescents is lacking (Gowers \& Bryant-Waugh, 2004); this has been highlighted in the National Institute for Health and Clinical Excellence (NICE) guidelines which underpin this evaluation (National Institute for Health and Clinical Excellence, 2004; Bryant-Waugh, 2006). In addition, mental health commissioners value user and carer participation across mental healthcare provision and this is reflected in the methodology of this study.

Structured programmes for anorexia have been discussed by several authors (Kaczynski et al, 2000; Anzai et al, 2002; Matsuvich et al, 2002; Halvorsen et al, 2004). These papers focused on describing the care provided or measuring outcome, but there is little in the literature on evaluation of the process of care. We report an evaluation of an in-patient treatment programme in the north of England.

\section{The Rainbow programme}

The Rainbow programme (developed by S.R. and A.B.) was introduced in January 2004 as a structured in-patient treatment programme for anorexia nervosa. Its four key aims are:

- to help the young person achieve healthy weight, maintain it and achieve balanced eating patterns;

- to help the young person with their distorted thoughts, including low self-esteem;

- to provide education and support for the family and young person and work collaboratively with them;

- to help the young person gain independence, and age-appropriate functioning.

The programme is structured by colour bands of the rainbow, which are determined by the weight: height ratio on admission. Weight: height ratio is one of the physical measures of anorexia and one of the aims of the programme is to achieve and maintain a healthy weight:height ratio. The Rainbow programme is set out in a user-friendly grid with a protocol for each colour band. The protocol defines the essential physical investigations and monitoring, meal plans and an individual therapeutic programme which includes individual therapy, a reintegration plan for education, a graded exercise plan and planned home leave. All families are offered family therapy and parent support groups throughout the young person's in-patient stay.

\section{Method}

There were two strands to the evaluation of the programme. The first was a notes review of a cohort of in-patients, collecting information against standards derived from the NICE guidelines on eating disorders (National Institute for Health and Clinical Excellence, 2004). The second strand was a series of questionnaires to users, carers and staff.

Eligibility criteria for inclusion were a period of in-patient care in the McGuiness Unit (a tertiary referral centre based in Manchester) and a diagnosis of anorexia nervosa. We identified 27 eligible patients via the electronic notes system, with admission dates from March 2003 to January 2006. Seventeen patients had been on the Rainbow programme and the remaining ten, whose admissions preceded the introduction of the programme, had been managed according to best practice prior to the introduction of the NICE guidelines.

The notes review was conducted during the spring of 2006 by E.R. and a clinical governance facilitator. The patient and carer questionnaires were mailed separately to 17 Rainbow programme families and the staff questionnaire was sent to all nursing and medical staff working on the in-patient unit on 1 December 2005; one reminder was sent to non-responders in all three groups within 2 months of the initial distribution. The two-tailed Fisher's exact test was used for statistical calculations.

\section{Results}

\section{Notes review}

There were two male patients in each group and the average age was slightly higher in the pre-Rainbow 
patients (184 v. 175 months). Male patients had shorter lengths of stay than female patients (136.5 v. 154 days),

original papers but length of stay did not vary significantly between the Rainbow and pre-Rainbow groups (152 v. 150 days). There was a higher proportion of patients with bradycardia in the Rainbow group (47 v. 10\%), which may suggest a more seriously affected group or may be due to improvements in recording.

The percentages of patients receiving appropriate assessment and service intervention were compared in each group (Table 1).

There was a statistically significant increase in the percentage of patients who received a routine blood screen, an electrocardiogram and a risk assessment in the Rainbow programme. There was a trend towards improvement in all assessment items, except for carers' assessments. There was an improvement in adherence to NICE guidelines. None of theses changes to service interventions reached statistical significance, but there is a trend towards improvement on the Rainbow programme.

\section{Service user and carer questionnaires}

The response rate for the user questionnaire was 8 out of $17(47 \%)$ and the response rate for the carer questionnaire was 5 out of 17 (29.4\%; selected responses to both questionnaires are shown in Table 2). There is scope for improvement in ensuring that all service users receive the Rainbow programme manual and that it is explained to them (for queries about the manual please contact Sandeep.Ranote@wwl.nhs.uk).

\section{Staff questionnaire}

The response rate to staff questionnaire was 17 out of 24 (70.8\%). Selected responses are shown in Table 3. Regular updating of the manual was suggested, with staff training.

\section{Discussion}

Our evaluation suggests that record keeping, physical assessment and access to service interventions improved following the introduction of the Rainbow programme. Our results are similar to a reported audit of NICE guidelines (Wessal, 2006), but more of our patients were offered family therapy (94 v. 44\% reported in Wessal, 2006). Despite the poor response rate from the carer questionnaires, carers gave more positive responses about the programme than users, perhaps reflecting a small number of motivated responders. The staff response rate was good at $70.8 \%$ and responders were generally positive about the programme. In particular, staff found it easier to formulate care plans after introduction of the Rainbow programme.

Evidence that structured programmes of care improve outcomes has been shown for a number of different disease areas, including fractured neck of the femur (Roberts et al, 2004) and palliative care
Table 1. Patients receiving appropriate assessment and service

intervention

\begin{tabular}{|c|c|c|}
\hline & $\begin{array}{c}\text { Rainbow } \\
\text { programme, } \\
n(\%)\end{array}$ & $\begin{array}{c}\text { Pre- } \\
\text { Rainbow, } \\
n(\%)\end{array}$ \\
\hline Physical exam completed & $16(94)$ & $8(80)$ \\
\hline Routine blood screen & $17(100)$ & $6(60)^{\star}$ \\
\hline Electrocardiogram & $13(77)$ & $2(20)^{* *}$ \\
\hline Risk assessment & $15(88)$ & $2(20)^{* *}$ \\
\hline Assessment by a psychologist & $4(24)$ & $1(10)$ \\
\hline Carer's assessment offered & $5(29)$ & $3(30)$ \\
\hline $\begin{array}{l}\text { Assessment of educational } \\
\text { needs }\end{array}$ & $11(64)$ & $4(40)$ \\
\hline Medication prescribed & $15(88)$ & $10(100)$ \\
\hline Antipsychotic or TCA & $5(29)$ & $3(30)$ \\
\hline Paediatric involvement & $13(77)$ & $4(40)$ \\
\hline NG tube used & $3(18)$ & $1(10)$ \\
\hline $\begin{array}{l}\text { Patients with NG tube treated } \\
\text { under Children's Act or } \mathrm{MHA}^{3}\end{array}$ & $3(100)$ & 0 \\
\hline Nutritional education offered ${ }^{3}$ & $16(94)$ & $7(70)$ \\
\hline Family therapy offered ${ }^{3}$ & $16(94)$ & $8(80)$ \\
\hline Individual therapy offered ${ }^{3}$ & $17(100)$ & $9(90)$ \\
\hline Art therapy offered & $11(65)$ & $8(80)$ \\
\hline Parent support group offered & $5(29)$ & 0 \\
\hline $\begin{array}{l}\text { TCA, tricyclic antidepressant; NG, nasogas } \\
\text { 1. } n=17 . \\
\text { 2. } n=10 \text {. } \\
\text { 3. NICE guideline (2004) recommendation. } \\
{ }^{\star} P<0.01 \text {, ** } P<0.001 \text {. }\end{array}$ & stric; MHA, Ment & Health Act. \\
\hline
\end{tabular}

Table 2. The use of the Rainbow Programme manual by service users and carers

\begin{tabular}{|lcc} 
& \multicolumn{2}{c}{ Positive responses, $n(\%)$} \\
\cline { 2 - 3 } Question & $\begin{array}{c}\text { Service users, } \\
n=8\end{array}$ & $\begin{array}{c}\text { Carers, } \\
n=5\end{array}$ \\
\hline Did you get a manual? & $5(62.5)$ & $4(80)$ \\
Was it explained? & $2(25)$ & $4(80)$ \\
Was it easy to use/follow? & $4(50)$ & $4(80)$ \\
\hline
\end{tabular}

Table 3. The staff questionnaire, selected responses

\begin{tabular}{lc} 
Question & $\begin{array}{c}\text { Positive } \\
\text { responses, } \\
n(\%)\end{array}$ \\
\hline $\begin{array}{l}\text { Did you find the grid a useful framework? } \\
\text { Was it easier to formulate individual care plans } \\
\text { from the grid? }\end{array}$ & $16(94)$ \\
$\begin{array}{l}\text { Were you able to use the 'band system' for } \\
\text { 'on-off activities' and 'home leave'? }\end{array}$ & $15(88)$ \\
Have you made use of the resources page? & $16(94)$ \\
Did you find the manual useful overall? & $12(71)$ \\
\hline
\end{tabular}

(Nightingale et al, 2003; Mirando et al, 2005). Improvements in the process of care such as record keeping and prescribing have been described in asthma and diabetes (Feder et al, 1995). Improvements in nursing skills following the introduction of protocols for diabetes 
have also been observed (Phillis-Tsimakas \& Walker, 2001).

\section{Limitations}

Limitations to our study include lack of randomisation, a small sample size and no measurement of outcomes. In addition, a number of the non-Rainbow patients had participated in a randomised study that was running in the unit, which may not have truly reflected 'treatment as usual'.

\section{Conclusions}

The evaluation showed that the Rainbow programme improved staff confidence in care planning. There was also evidence of improvements in the process of assessment and record keeping.

\section{Declaration of interest}

None.

\section{References}

ANZAI, N., DUDLEY, L. \& BIDWELL, R (2002) In-patient and partial hospital treatment for adolescent eating disorders. Child and Adolescent Psychiatric Clinics of North America, 11, 279-309.

BRYANT-WAUGH, R. (2006) Recent developments in anorexia nervosa.

Child and Adolescent Mental Health, 11, $76-81$

FEDER, G., GRIFFITHS, C. \& HIGHTON, C. (1995) Do clinical guidelines introduced with practice-based education improve care of asthmatic and diabetic patients? BMJ, 311, 1473-1478.
GOWERS, S. \& BRYANT-WAUGH, R. (2004) Management of child and adolescent eating disorders: the current evidence base and future directions. Journal of Child Psychology and Psychiatry, 45, 63-83.

GOWERS, S. G., WEETMAN, J., SHORE, A., et al (2000) Impact of hospitalisation on the outcome of adolescent anorexia nervosa. British Journal of Psychiatry, 176, 138-141.

HALVORSEN, I., ANDERSON, A. \& HEYERDAHL, S. (2004) Good outcome of adolescent onset anorexia nervosa after systematic treatment.

Intermediate to long-term follow-up of a representative county sample. European Child and Adolescent Psychiatry, 13, 295-306.

KACZYNSKI, J., DENISON, H., WIKNERTZ, A., et al (2000) Structured care programme yielded good results in severe anorexia nervosa. Lakartidningen, 97, 2734-2737.

MATSUVICH, D., GARCIA, A., GUTT, S. et al (2002) Hospitalisation of patients with anorexia nervosa: a therapeutic proposal. Eating and Weight Disorders, 7,196-201.

MIRANDO, S., DAVIES, P. D. \& LIPP, A. (2005) Introducing an integrated care

pathway for the last days of life. Palliative Care Medicine, 19, 351-352. NATIONAL INSTITUTE FOR HEALTH AND CLINICAL EXCELLENCE (2004) Eating Disorders: Core Interventions in the Treatment and Management of

Anorexia Nervosa, Bulimia and Related Eating Disorders. NICE.

NIGHTINGALE, E., KRISTJANSON, L. \&

TOYE, C. (2003) Evaluating the navigate care model: clinical palliative care pathways based on anticipated care outcomes International Journal of Palliative Nursing, 9, 298-307.

PHILLIS-TSIMAKAS, A \& WALKER, C (2001) Improved care of diabetes in underserved populations. Journal of Ambulatory Care Management, 24, $39-43$.

ROBERTS, H., PICKERING, R., ONSLOW E., et al (2004) The effectiveness of implementing a care pathway for femoral neck fracture in older people: a prospective controlled before and after study. Age and Ageing, 33 , $178-184$

WESSAL, A. (2006) How a child and adolescent service manages anorexia nervosa. Advances in Psychiatric Treatment, e-letter (http:// apt.rcpsych.org/cgi/eletters/12/ $1 / 5)$

*Elizabeth Rous Consultant Child and Adolescent Psychiatrist, Stockport CAMHS Pennine CareTrust, Tree House Children's Centre, Stepping Hill Hospital, Poplar Grove, Stockport SK2 7JE, email: libby.rous@nhs.net,

Sandeep Ranote Consultant Child and Adolescent Psychiatrist, Wigan CAMHS (Eating disorders Lead), 5 Boroughs Partnership NHS Trust, Rachel Elvins Clinical Tutor and Honorary Specialist Registrar, University of Manchester, Asha Bhatt Consultant Child and Adolescent Psychiatrist, Bolton Salford and Trafford Mental Health NHS Trust original

papers 